the colon with a long tube. Sugar, starch and fat can be done with only a small mortality. We are should be given in very small quantity, because of told that the uterus has no function after the removal their tendency to fermentation. The food should of the appendages, but this has not been demonstrated; consist chiefly of tender meat well minced, toasted and, on the contrary, we know that the sexual life of bread, milk, soft-boiled eggs, oysters and concentrated the woman is very much better preserved by leaving broths. As improvement appears a larger variety may the uterus, and that the mental effect is also much be gradually introduced.

In the administration of medicine it is necessary to provide for an increase of motor and digestive power while endeavoring to prevent the process of fermentation in the gastric contents. For the first object, one-fiftieth of a grain of nitrate of strychnin may be injected hypodermically every six hours. Ten drops of dilute hydrochloric acid should be taken in four ounces of hot water after the three principal meals each day. One-half hour before each of those meals the patient should take five grains of salol or of salicylic acid, or a grain of resorcin or of carbolic acid, or five grains of sodium hyposulphite, to check the fermentative process during the approaching period of attempted digestion. As soon as the patient begins to feel able to remain for a considerable time in the open air the anemia that is present may be controlled with large doses of carbonate of iron.

Recently, the aid of surgery has been invoked with some degree of success in the relief of those forms of gastric dilatation that are dependent upon stenosis of the pylorus. Notwithstanding the high degree of mortality, it is sometimes the only resource, and, fortunately, with increasing experience the death rate is slightly diminished. In certain obstinate cases without pyloric obstruction, the operation of folding the anterior wall of the stomach upon itself and sewing the border of the greatest curvature to the margin of the upper curve of the organ-just as a sailor takes a reef in a sail-has been performed with tolerable success.

\title{
TREATMENT OF PERIUTERINE SEPTIC DISEASES.
}

Read at the Richmond meeting of the American Association of Obstetricians and Gyuecologists.

BY W. E. B. DAVIS, M.D.

PROFFSSOR OF GYNECOLOGY AND ABDOMINAL SURGERY, BIRMINGHAM MEDICAL COLLEGE, MIRMINGHAM, ALA.

Only recently has the extremely radical procedure of hysterectomy been practiced in this country for septic diseases of the internal genitals. A wave, which had its origin in Paris at the hands of Péan, aided by Richelot, Segond, Jacobs and others, reached our shores three years ago and has found a considerable following among our leading operators. There has been much rivalry for some years in this country as to who could do the largest number of hysterectomies most successfully, but I will not say that it is possible this innovation was more readily embraced as it afforded great opportunity for such ambitious ones, who had almost ceased to be interested in the more simple operation of salpingo-oöphorectomy, to increase their cases of hysterectomy more rapidly. They claim that there is no use in leaving the uterus behind after the removal of the appendages; that "in every operation for septic diseases of the generative organs which demands the removal of the tubes and ovaries hysterectomy should also be performed, unless there are plain contradictions forbidding it."

It should be the aim and pride of the surgeon to preserve everything consistent with thorough surgical th work, and not to sacrifice important organs because it

better. A slow convalescence, or even a second operation, is preferable to its removal, unless very much diseased. It is a reflection on the correctness of the reports of complete recoveries of such a large per cent. of cases by many most excellent surgeons, when the uterus was not removed, to accept the argument now being used in favor of hysterectomy in all these cases. As stated at the last meeting of the AMERICAN Medical Association, "I can not agree with Dr. Sutton and others that pus in the tubes is due to gonorrhea in 75 per cent. of cases. I think that puerperal infection is the cause of more than 50 per cent. Tubercular infection is rarely the cause, and is not so important as has been claimed. However, the importance which has been attached to gonorrhea is against the argument for the removal of the uterus, as the infection from this source is not deep and can be removed with the curette. Because some cases are not completely cured by the removal of the appendages is no argument for hysterectomy in every case where the bilateral operation is required, for nearly all these can be relieved by a thorough curettage. Some large uteri will require, in addition to this, a high amputation of the cervix. Only a small number will need hysterectomy."

It is a notable fact that some of the surgeons who claimed most for curettment of the uterus a few years ago and who taught that total ablation of the appendages was seldom necessary are now the most enthusiastic advocates of hysterectomy. It is granted that there are some cases in which it is best to remove the uterus, those in which the uterus is flaccid and its walls intiltrated with pus, surrounded with pus tubes and ovarian abscesses. However, such cases are rare. Tubercular infection of the uterus, too, is sufficient cause to require its removal. Extreme injury and mutilation of the organ in removing the tubes and ovaries would also call for the operation. Vaginal incision for the drainage of pus in the pelvis not confined to the tubes and ovaries has been practiced for a long time with very gratifying results. This procedure is so old that it is hard to say when it was not resorted to. Such cases would recover, almost without exception, from the immediate attack and a large number recovered completely and bore children afterward. This result was due to the drainage of the tube or ovary through the peritubal and periovarian abscess, just as cases of appendicitis are cured by the rupture of the diseased appendix producing an abscess, which is evacuated by the surgeon, the appendix being drained through the abscess. In cases of abscess from lymphangitis and phlebitis this treatment was all that was required, but cases of abscess due to the tube or ovary were not all entirely relieved, due to the fact that the pus tube or ovarian abscess was not drained satisfactorily.

More recently pus tubes and ovarian abscesses have been incised and drained through the vagina with permanent recoveries in a good proportion of cases. These are the very cases where the vaginal operation and hysterectomy have been commended so highly by the French surgeons. Yet a large per cent. of these cases can be relieved by vaginal incision and drain- 
age. If not relieved the patient's condition will be made better by getting rid of the pus, and later on an abdominal operation can be done and the patient cured by the removal of the appendages-and perhaps of one side only.

The French surgeons, in some cases, when unable to remove the diseased tubes and ovaries after hysterectomy, have been contented to open the masses containing the diseased appendages and to pack with gauze. The drainage in such cases has brought about complete cures. The same result could have been accomplished without the removal of the uterus, by vaginal section and the breaking up of adhesions with drainage. I would not be misunderstood as believing that all cases can be cured by incision and drainage, but so large a per cent. can be relieved, without the loss of the uterus and adnexa, our cases should be given the benefit of this conservative procedure before more radical measures are resorted to. Especially should this be done in young women, to whom the loss of the appendages and uterus means the loss of so much that is necessary to their happiness. This treatment has been highly commended by Henrotin, Pryor and Kelly, and my own experience with it, which is limited, has been so satisfactory that I am convinced of its great value and the necessity of its more general adoption. The following is the plan I have adopted:

Peritubal and periovarian abscesses.-When there is a considerable collection of pus and the patient is suffering from marked sepsis the uterus is not curetted and no attempt is made to find the tube or ovary. An incision is made into the absccss, hugging the posterio-uterine wall, and after thorough irrigation gauze is packed in the cavity, the vaginal incision being well filled so as to keep a large opening to allow the gradual withdrawal of the gauze-permitting of the escape of any collection that may occur in the cavity. The vagina is also filled with sterilized gauze. The gauze in the vagina is removed in twenty-four hours and from the abscess cavity in forty-eight hours. A rubber tube is introduced into the abscess cavity at the end of a week and kept in place from four to eight weeks. Douches are given twice a day after the removal of the gauze from the vagina. Water is passed through the tube twice a day. Carbolic acid may be used with advantage in the wash. In small peritubal and periovarian abscesses the same treat. ment should be adopted as in cases of tubal and ovarian abscesses, which I will describe. If there is a considerable mass some weeks after the drainage of large pelvic abscesses (peritubal and periovarian abscesses) the same treatment will be indicated as for tubal and ovarian abscesses.

Tubal and ouchicm abscesses. - In these cases the uterus is always curetted and pure carbolic acid applied to the cavity. The cervix is grasped by a strong vulsellum forceps and pulled down and lifted to the front. An incision, with scissors, is made in the posterior vaginal wall, hugging the cervix and large enough to admit two fingers. When the peritoneum is reached the mass or masses can be easily outlined by bimanual palpation and can be opened with scissors or uterine forceps, the finger being used as a guide. The cavity is thoroughly dried with gauze and irrigated. If the general abdominal cavity is entered it is best to make the opening large so as to prevent a secondary abscess. Gauze should be packed into the tube and extend into the abdominal cavity, a one pill daily for two days he was so much improved large quantity being used in the vaginal incision. The vagina should also be packed with gauze. The gauze is removed from the vagina in forty-eight hours, and from the abdomen and tube in five or six days, except the withdrawal of a few inches each day to keep the vaginal opening patent for the escape of fluid. A rubber tube is introduced after the removal of the gauze and managed as directed for peritubal abscess. As Henrotin says: "It seems only reasonable that such operations may be successful, even in the most chronic forms of pyosalpinx. What happens in such cases? Septic material traveling up the Fallopian tube, when the process is not too rapid, finds itself arrested at the abdominal opening by a closure of the ostium abdominale. An abscess cavity is developed in the walls of the tube proper. After a time the uterine end becomes closed, and tinen we have independent pus sac, not communicating with either the serous or the uterine cavity. This may rupture into the fold of the broad ligament and work its way toward the vagina. If widely opened and drained and packed, after a time all the so-called pyogenic lining membrane disappears, granulations fill the gap, the Fallopian tube becomes obsolete and the patient is cured."

Hydrosalpinx and hematosalpinx should be dealt with as outlined for pyosalpinx. I have treated some cases of ectopic gestation by this method, and was recently consulted by a young woman who had become pregnant in the uterus after recovery from such an operation for pregnancy in the right tube.

Pelvic masses following an abortion or delivery at term should be treated in this way. Pus should not be waited for. I have treated a good number of such cases by incision and gauze packing before pus formation, and thus cut short a protracted illness.

I have operated a few times in cases where the uterus was retrodisplaced and bound down by adhesions. The adhesions were broken up, the uterus replaced, the tubes and ovaries lifted up and gauze packing resorted to as heretofore described. The results so far have been quite satisfactory, but I am of the opinion that a large number of such cases will require salpingo-oöphorectomy before they are entirely relieved.

When double salpingo-oöphorectomy is necessary the choice of operation will depend largely upon the decision as to the disposition of the uterus. If the uterus is to be removed, in such cases the vaginal route could be adopted with advantage in a good proportion of operations. Fortunately that is not necessary and hence the abdominal operation is preferable, "as the aid of positjve sight is given, thus affording a wider field of operation and an opportunity for conservatism."

\section{EFFECTIVE REMEDIES IN TYPHOID FEVER.}

BY THOMAS B. HEIMSTREET, M.D. TROY, N. Y.

About five years ago, being called to a patient who had prodromic symptoms of typhoid fover, and insisted on some prompt remedy, I gave him a half. grain pill of hydrarg. protiodid, knowing it to be one of the most powerful antiseptics and having frequently prescribed it in cases of specific disease, for several days 